\title{
BIRTH WEIGHTS OF SOUTH AFRICAN BABIES
}

\author{
BY \\ EVA J. SALBER* and EVELYN S. BRADSHAW \\ Institute of Family and Community Health, Union Health Department, Durban, \\ Union of South Africa
}

This present paper is a preliminary report on the birth weights and the incidence of prematurity in groups of South African babies, with special reference to race and sex. Other factors affecting birth weight (economic status as reflected by the hospital class, birth rank, age of mother, and season of birth) are being investigated and will be reported subsequently.

\section{MATERIAL AND METHOD}

The data were collected from various hospitals and nursing homes in Durban, Pietermaritzburg, and Capetown:

European babies from two private nursing homes in Durban.

Coloured (i.e. half-caste) babies from two provincial hospitals, one in Pietermaritzburg and one in Capetown.

Bantu babies from a provincial hospital in Pietermaritzburg, and a mission hospital in Durban.

Indian babies from a provincial hospital in Pietermaritzburg, a mission hospital in Durban and a government aided hospital in Durban.

The data extracted consisted of birth weight, race of mother, and sex of child. Abortions and multiple births were excluded. Frequency distributions of the birth weights. were drawn up for the different races and sexes, and the incidence of prematurity (birth weight less than $5 \mathrm{lb} .8 \mathrm{oz}$.) was determined.

\section{RESULTS}

RACE AND BIRTH Weight.-The distributions of birth weights for the four groups are given in the Appendix (see also the Figure, overleaf), and the mean birth weights and differences between the means are shown in Tables IA, IB, IC (overleaf). There are substantial differences between mean birth weights:

$$
\begin{array}{ll}
\text { European } 7.47 \mathrm{lb} . & \text { Bantu } 6.77 \mathrm{lb} \text {. } \\
\text { Coloured } 6.85 \mathrm{lb} . & \text { Indian } 6.46 \mathrm{lb} \text {. }
\end{array}
$$

In relation to these differences it should be noted that the kinds of persons delivered in institutions vary between the four groups. In Durban it is usual for European mothers (at all birth ranks) to have their babies in hospital, but institutional deliveries are more common for first births among Non-European mothers.

* Senior Bursar, Council for Scientific and Industrial Research. 


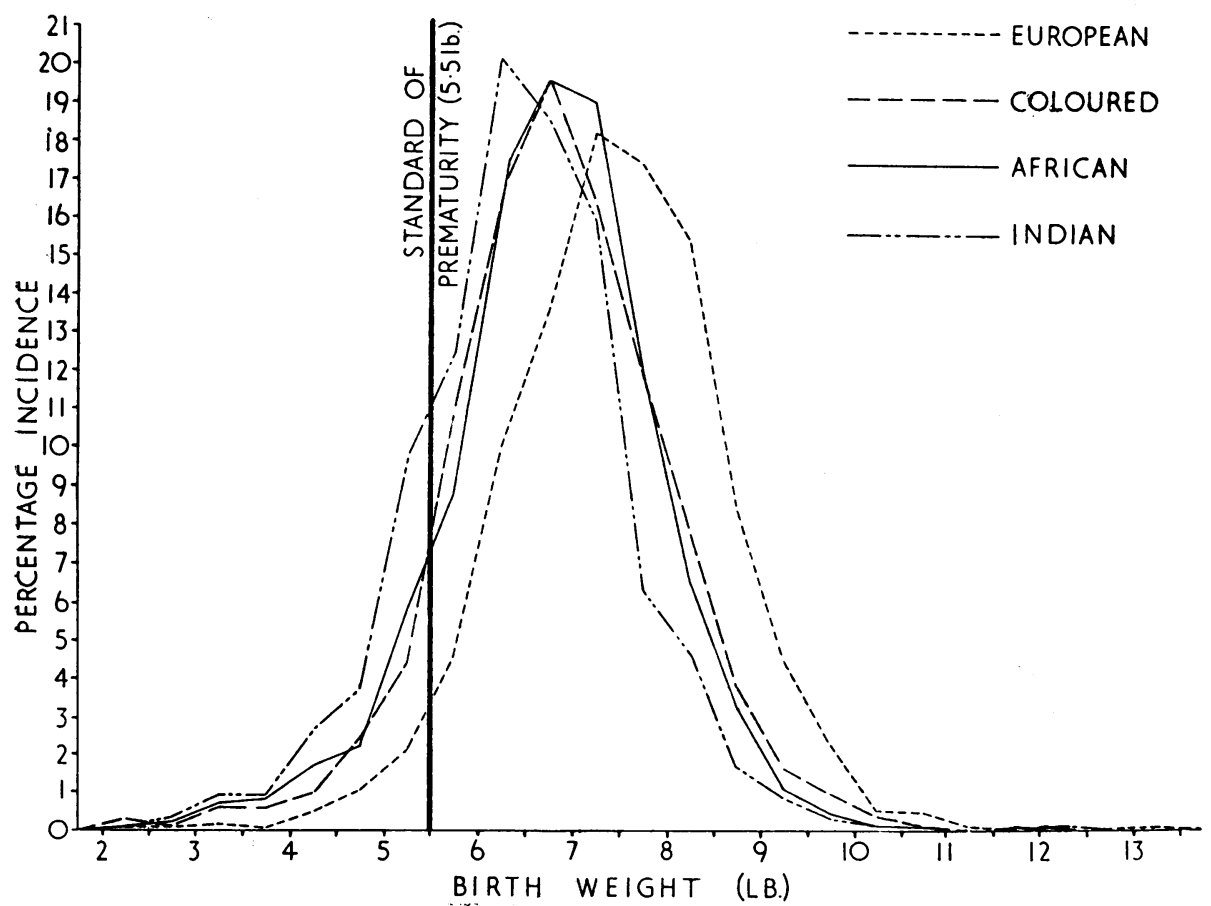

FiguRE.-Frequency curves of birth weights of different racial groups (sexes combined).

On the other hand there are more babies of higher birth order among Non-Europeans. If, as is generally believed, first babies are lighter than later births, these differences may be expected to influence the mean birth weights.

SEX AND Birth Weight.-Analysis of birth weights of boys and girls in the four groups showed that:

(i) in each group boys have a higher mean birth weight than girls;

(ii) differences between the birth weights of boys and of girls as between the groups are similar to those observed when the sexes are combined (Tables IA, IB, IC).

With one exception (Coloured) these differences are all statistically significant.

InCIDENCE of Prematurity.-Births are here considered to be premature if they weighed less than $5 \mathrm{lb} .8 \mathrm{oz}$. The incidence of prematurity in each group was:

European $4 \cdot 2$ per cent.

Coloured 9.6 per cent.

Bantu $11 \cdot 5$ per cent.
Indian $18 \cdot 3$ per cent. Tables IIA, IIB, IIC overleaf.

As might be expected, the incidence of prematurity is inversely related to the mean birth weight, and is in consequence somewhat higher for girls than for boys. With one exception (as between Coloured and Bantu) the differences between the four groups are all statistically significant.

It may be noted that the proportion of birth weights recorded was not the same 
TABLE IA

Mean Birth Weight related to Sex and Race

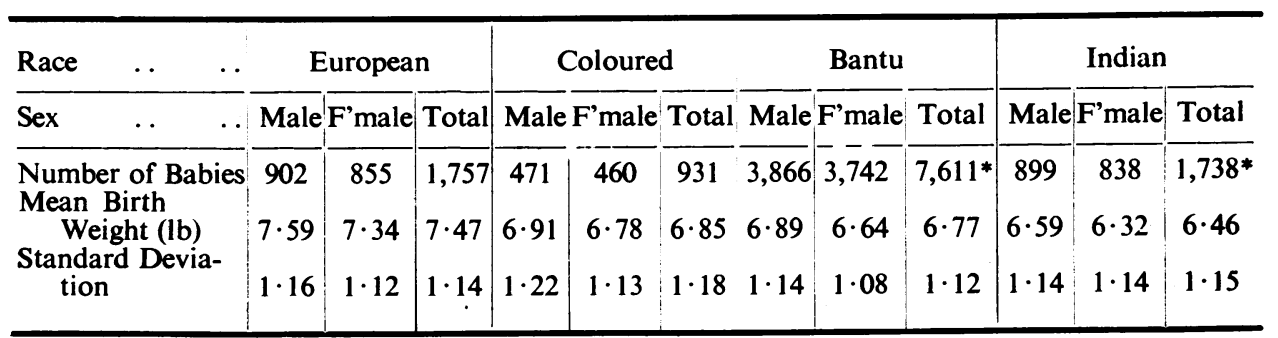

* Including 3 Bantu and 1 Indian of unspecified sex.

TABLE IB

Difference in Mean Weight by Race for Each Sex .

\begin{tabular}{|c|c|c|c|c|c|c|c|c|c|c|}
\hline \multirow[b]{2}{*}{ Difference } & \multirow[b]{2}{*}{ Between } & \multicolumn{3}{|c|}{ Male } & \multicolumn{3}{|c|}{ Female } & \multicolumn{3}{|c|}{ Total } \\
\hline & & $\begin{array}{l}(a) \\
\text { Differ- } \\
\text { ence } \\
\text { (lb.) }\end{array}$ & $\begin{array}{l}(b) \\
\text { Stan- } \\
\text { dard } \\
\text { error } \\
\text { of } \\
\text { differ- } \\
\text { ence }\end{array}$ & $(a) \div(b)$ & $\begin{array}{l}(a) \\
\text { Differ- } \\
\text { ence } \\
\text { (lb.) }\end{array}$ & $\begin{array}{l}(b) \\
\text { Stan- } \\
\text { dard } \\
\text { error } \\
\text { of } \\
\text { differ- } \\
\text { ence }\end{array}$ & $(a) \div(b)$ & $\begin{array}{c}(a) \\
\text { Differ- } \\
\text { ence } \\
\text { (lb.) }\end{array}$ & $\begin{array}{l}\text { (b) } \\
\text { Stan- } \\
\text { dard } \\
\text { error } \\
\text { of } \\
\text { differ- } \\
\text { ence }\end{array}$ & $(a) \div(b)$ \\
\hline $\begin{array}{l}\text { European } \\
\text { and }\end{array}$ & $\begin{array}{l}\text { Coloured } \\
\text { Bantu } \\
\text { Indian }\end{array}$ & $\begin{array}{l}0 \cdot 68 \\
0 \cdot 70 \\
1 \cdot 00\end{array}$ & $\begin{array}{l}0.069 \\
0.042 \\
0.054\end{array}$ & $\begin{array}{r}9 \cdot 9 \\
16 \cdot 7 \\
18 \cdot 5\end{array}$ & $\begin{array}{l}0.56 \\
0 \cdot 70 \\
1 \cdot 02\end{array}$ & $\begin{array}{l}0.065 \\
0.042 \\
0.055\end{array}$ & $\begin{array}{r}8 \cdot 6 \\
16 \cdot 7 \\
18 \cdot 5\end{array}$ & $\begin{array}{l}0.62 \\
0.70 \\
1.01\end{array}$ & $\begin{array}{l}0.047 \\
0.030 \\
0.039\end{array}$ & $\begin{array}{l}13 \cdot 2 \\
23 \cdot 3 \\
25 \cdot 9\end{array}$ \\
\hline $\begin{array}{c}\text { Coloured } \\
\text { and }\end{array}$ & $\begin{array}{l}\text { Bantu } \\
\text { Indian }\end{array}$ & $\begin{array}{l}0.02 \\
0 \cdot 32\end{array}$ & $\begin{array}{l}0.059 \\
0.068\end{array}$ & $\begin{array}{l}0 \cdot 3 \\
4 \cdot 7\end{array}$ & $\begin{array}{l}0 \cdot 14 \\
0 \cdot 46\end{array}$ & $\begin{array}{l}0.056 \\
0.066\end{array}$ & $\begin{array}{l}2 \cdot 5 \\
7 \cdot 0\end{array}$ & $\begin{array}{l}0 \cdot 08 \\
0 \cdot 39\end{array}$ & $\begin{array}{l}0.041 \\
0.047\end{array}$ & $\begin{array}{l}2 \cdot 0 \\
8 \cdot 3\end{array}$ \\
\hline Bantu and & Indian & $0 \cdot 30$ & 0.042 & $7 \cdot 1$ & 0.32 & 0.043 & $7 \cdot 4$ & $0 \cdot 31$ & 0.031 & $10 \cdot 0$ \\
\hline
\end{tabular}

TABLE IC

Differences in Mean Weight by Sex for Each Race

\begin{tabular}{|c|c|c|c|c|c|c|}
\hline & Race & & & $\begin{array}{l}(a) \\
\text { Difference } \\
\quad(\text { lb. })\end{array}$ & $\begin{array}{c}(b) \\
\text { Standard Error } \\
\text { of Difference }\end{array}$ & $(a) \div(b)$ \\
\hline $\begin{array}{l}\text { European } \\
\text { Coloured } \\
\text { Bantu } \\
\text { Indian }\end{array}$ & $\begin{array}{l}\ldots \\
\ldots \\
\ldots \\
\ldots\end{array}$ & $\begin{array}{l}\ldots \\
\ldots \\
\ldots \\
\ldots\end{array}$ & $\begin{array}{l}\ldots \\
\ldots \\
\ldots \\
\ldots\end{array}$ & $\begin{array}{l}0 \cdot 25 \\
0 \cdot 13 \\
0 \cdot 25 \\
0 \cdot 27\end{array}$ & $\begin{array}{l}0.054 \\
0.077 \\
0.026 \\
0.055\end{array}$ & $\begin{array}{l}4 \cdot 6 \\
1 \cdot 7 \\
9 \cdot 6 \\
4 \cdot 9\end{array}$ \\
\hline
\end{tabular}

in every hospital, and in one case (an Indian hospital) 23 per cent. of births were not weighed in 1948. Infants are sometimes shown as "premature" without the actual weight being given, and it is therefore possible that the incidence of prematurity is higher than our figures indicate. 
TABLE IIA

Percentage of Premature Bábieś (less than $5 \cdot 5$ lb.) by Sex for Each Race

\begin{tabular}{|c|c|c|c|c|c|c|c|c|c|c|c|c|}
\hline Race & & Europear & & & Coloure & & & Bantu & & & Indian & \\
\hline $\begin{array}{ll}\cdots & \ldots\end{array}$ & Male & F'male & Total & Male & F'male & Total & Male & F'male & Total & Male & F'male & e Total \\
\hline $\begin{array}{l}\text { Number of } \\
\text { babies weighed } \\
\text { Percentage less } \\
\text { than } 5.5 \mathrm{lb} .\end{array}$ & $\begin{array}{l}902 \\
3 \cdot 8\end{array}$ & $\begin{array}{l}855 \\
4 \cdot 6\end{array}$ & $\mid$\begin{tabular}{c|}
1,757 \\
$4 \cdot 2$
\end{tabular} & $\begin{array}{l}471 \\
9 \cdot 6\end{array}$ & $\begin{array}{l}460 \\
9 \cdot 6\end{array}$ & $\begin{array}{l}931 \\
9 \cdot 6\end{array}$ & $\begin{array}{l}3,866 \\
10 \cdot 3\end{array}$ & $\begin{array}{r}3,742 \\
12 \cdot 9\end{array}$ & $\begin{array}{l}7,611 * \\
11 \cdot 5\end{array}$ & $\begin{array}{l}899 \\
14 \cdot 2\end{array}$ & $\begin{array}{r}838 \\
22 \cdot 7\end{array}$ & $\begin{array}{l}1,738^{*} \\
18 \cdot 3\end{array}$ \\
\hline
\end{tabular}

* Includes 3 Bantu and 1 Indian of unspecified sex.

TABLE IIB

Differences in Percentage of Premature Babies between Races for Each Sex

\begin{tabular}{|c|c|c|c|c|c|c|c|c|c|c|}
\hline \multirow{2}{*}{$\begin{array}{l}\text { Sex } \quad \cdots \\
\text { Difference }\end{array}$} & \multirow{2}{*}{$\frac{\cdots}{\text { Between }}$} & \multicolumn{3}{|c|}{ Male } & \multicolumn{3}{|c|}{ Female } & \multicolumn{3}{|c|}{ Total } \\
\hline & & $\begin{array}{c}(a) \\
\text { Differ- } \\
\text { ence } \\
\text { (per } \\
\text { cent.) }\end{array}$ & $\begin{array}{l}(b) \\
\text { Stan- } \\
\text { dard } \\
\text { error } \\
\text { of } \\
\text { differ- } \\
\text { ence }\end{array}$ & $(a) \div(b)$ & $\begin{array}{c}(a) \\
\text { Differ- } \\
\text { ence } \\
\text { (per } \\
\text { cent.) }\end{array}$ & $\begin{array}{l}(b) \\
\text { Stan- } \\
\text { dard } \\
\text { error } \\
\text { of } \\
\text { differ- } \\
\text { ence }\end{array}$ & $(a) \div(b)$ & $\begin{array}{c}(a) \\
\text { Differ- } \\
\text { ence } \\
\text { (per } \\
\text { cent.) }\end{array}$ & $\begin{array}{l}(b) \\
\text { Stan- } \\
\text { dard } \\
\text { error } \\
\text { of } \\
\text { differ- } \\
\text { ence }\end{array}$ & $(a) \vdots(b)$ \\
\hline $\begin{array}{l}\text { European } \\
\text { and }\end{array}$ & $\begin{array}{l}\text { Coloured } \\
\text { Bantu } \\
\text { Indian }\end{array}$ & $\begin{array}{r}5 \cdot 8 \\
6 \cdot 5 \\
10 \cdot 4\end{array}$ & $\begin{array}{l}1 \cdot 50 \\
0 \cdot 80 \\
1 \cdot 33\end{array}$ & $\begin{array}{l}3 \cdot 9 \\
8 \cdot 1 \\
7 \cdot 8\end{array}$ & \begin{tabular}{r|}
$5 \cdot 0$ \\
$8 \cdot 3$ \\
$18 \cdot 1$
\end{tabular} & $\begin{array}{l}1.55 \\
0.90 \\
1 \cdot 61\end{array}$ & $\begin{array}{r}3 \cdot 2 \\
9 \cdot 2 \\
11 \cdot 2\end{array}$ & \begin{tabular}{r|}
$5 \cdot 4$ \\
$7 \cdot 3$ \\
$14 \cdot 1$
\end{tabular} & $\begin{array}{l}1 \cdot 08 \\
0 \cdot 60 \\
1 \cdot 04\end{array}$ & $\begin{array}{r}5 \cdot 0 \\
12 \cdot 2 \\
13 \cdot 6\end{array}$ \\
\hline $\begin{array}{l}\text { Coloured } \\
\text { and }\end{array}$ & $\begin{array}{l}\text { Bantu } \\
\text { Indian }\end{array}$ & $\begin{array}{l}0 \cdot 7 \\
4 \cdot 6\end{array}$ & $\begin{array}{l}1 \cdot 44 \\
1 \cdot 79\end{array}$ & $\begin{array}{l}0.5 \\
2.6\end{array}$ & $\begin{array}{r}3 \cdot 3 \\
13 \cdot 1\end{array}$ & $\begin{array}{l}1 \cdot 48 \\
2 \cdot 00\end{array}$ & $\begin{array}{l}2 \cdot 2 \\
6 \cdot 6\end{array}$ & $\begin{array}{l}1 \cdot 9 \\
8 \cdot 7\end{array}$ & $\begin{array}{l}1 \cdot 03 \\
1 \cdot 34\end{array}$ & $\begin{array}{l}1 \cdot 8 \\
6 \cdot 5\end{array}$ \\
\hline Bantu and & Indian & $3 \cdot 9$ & $1 \cdot 26$ & $3 \cdot 1$ & $9 \cdot 8$ & $1 \cdot 55$ & $6 \cdot 3$ & $6 \cdot 8$ & $1 \cdot 00$ & $6 \cdot 8$ \\
\hline
\end{tabular}

TABLE IIC

Differences in Percentage of Premature Babies between Sexes by Race

\begin{tabular}{|c|c|c|c|c|c|c|}
\hline & Race & & & $\begin{array}{c}(a) \\
\text { Difference } \\
\text { (per cent.) }\end{array}$ & $\begin{array}{l}\text { (b) } \\
\text { Standard Error } \\
\text { of Difference }\end{array}$ & $(a) \div(b)$ \\
\hline $\begin{array}{l}\text { European } \\
\text { Coloured } \\
\text { Bantu } \\
\text { Indian }\end{array}$ & $\begin{array}{l}\cdots \\
\cdots \\
\cdots\end{array}$ & $\begin{array}{l}\cdots \\
\cdots \\
\cdots \\
\cdots\end{array}$ & $\begin{array}{l}\cdots \\
\cdots \\
\cdots \\
\cdots\end{array}$ & $\begin{array}{l}0 \cdot 8 \\
0 \cdot 0 \\
2 \cdot 6 \\
8 \cdot 5\end{array}$ & $\begin{array}{l}0.96 \\
1.93 \\
0.73 \\
1 \cdot 86\end{array}$ & $\begin{array}{l}0 \cdot 8 \\
0 \cdot 0 \\
3 \cdot 6 \\
4 \cdot 6\end{array}$ \\
\hline
\end{tabular}

\section{Discussion}

Our data on mean birth weight for four racial groups may be compared with the observations by other workers which are set out in Table III (opposite).

In general mean birth weights are higher for Europeans than for Non-Europeans. In America it has been reported that white children are heavier at birth than Negro children (Bakwin, 1932; Bivings, 1934; Michelson, 1943), and it has been suggested 
TABLE III

Particulars of Previous Investigations

\begin{tabular}{|c|c|c|c|c|c|}
\hline Investigation & Date & Numbers & Race & Place of Birth & $\begin{array}{l}\text { Mean Birth } \\
\text { Weight (lb.) }\end{array}$ \\
\hline Le Riche & 1938 & 942 & European & $\begin{array}{l}\text { Moedersbond Hos- } \\
\text { pital, Praetoria }\end{array}$ & $7 \cdot 46$ \\
\hline \multirow[t]{2}{*}{$\begin{array}{c}\text { Cape Coloured Com- } \\
\text { mission }\end{array}$} & 1937 & 906 & European & $\begin{array}{l}\text { Peninsula Maternity } \\
\text { Hospital, Cape- } \\
\text { town }\end{array}$ & $7 \cdot 42$ \\
\hline & & 1,763 & $\begin{array}{c}\text { Non-European } \\
\text { (mainly } \\
\text { Coloured) }\end{array}$ & - & $7 \cdot 01$ \\
\hline \multirow[t]{3}{*}{ Heyns and Hersch } & 1944 & 1,216 & Bantu & $\begin{array}{l}\text { Bridgeman Memorial } \\
\text { Hospital, Johan- } \\
\text { nesbeirg }\end{array}$ & $6 \cdot 74$ \\
\hline & & 967 & Bantu & $\begin{array}{l}\text { King Edward Hos- } \\
\text { pital, Durban }\end{array}$ & $6 \cdot 82$ \\
\hline & & 440 & Bantu & $\begin{array}{l}\text { University Clinic, } \\
\text { Alexandra, } \\
\text { Johannesburg }\end{array}$ & $7 \cdot 28$ \\
\hline
\end{tabular}

that differences in economic status may have an important bearing on this observation. The same explanation may be relevant in the case of the four groups here recorded, since gross differences in net income are exhibited in Table IV (prepared from the Natal Regional Survey*).

TABLE IV

INCOMES OF DIFFERENT RACES

\begin{tabular}{|c|c|c|c|c|}
\hline Race & European & Coloured & African & Indian \\
\hline Number of households investigated & 711 & 68 & 493 & 497 \\
\hline Average number of persons & $3 \cdot 9$ & $4 \cdot 4$ & $3 \cdot 1$ & $6 \cdot 0$ \\
\hline Average net available weekly income & f7 $19 \quad 9$ & $£ 3 \quad 18 \quad 7$ & $£ 179$ & $£ 2 \quad 13 \quad 0$ \\
\hline
\end{tabular}

The sex differences in mean birth weight are also in accord with American reports (Meredith and Brown, 1939; Palmer and Ciocco, 1945). Jeans and Marriott (1947) attribute the weight difference to the difference in height and suggest that for infants of the same height there is little difference between the sexes.

The incidence of prematurity here recorded for European births, and the sex difference in incidence, are consistent with English and American reports (Dunham, Jenss, and Christie, 1939; Stevenson, 1950). Again, it seems reasonable to assume that differences in economic status have a considerable bearing on the differences in the incidence of prematurity exhibited by the four groups.

\footnotetext{
* Personal communication from Professor Burrows .Department of Economics, Natal University.
} 


\section{SUMMARY}

Birth weights of infants (1,757 European, 931 Coloured, 7,611 Bantu, and 1,738 Indian) were studied in relation to race, sex, and incidence of prematurity, and the following results obtained:

(1) Race.-Mean birth weights for the four groups:

$$
\begin{array}{llll}
\text { European } & 7.47 \mathrm{lb} . & \text { Bantu } & 6.77 \mathrm{lb} \text {. } \\
\text { Coloured } 6.85 \mathrm{lb} . & \text { Indian } & 6.46 \mathrm{lb} \text {. }
\end{array}
$$

It is suggested that the observed differences may be explained by differences in economic status of the parents.

(2) Sex.-Mean birth weights in each racial group were higher for males than for females. The racial differences in mean birth weights already noted are again observed when the sexes are considered separately.

(3) Prematurity.-The incidence of prematurity (birth weight less than $5 \mathrm{lb} .8 \mathrm{oz}$.) in each racial group was:

$$
\begin{aligned}
& \text { European } 4 \cdot 2 \text { per cent. Bantu } 11.5 \text { per cent. } \\
& \text { Coloured } 9.6 \text { per cent. Indian } 18.3 \text { per cent. }
\end{aligned}
$$

The incidence of prematurity was higher for females than for males, as might be anticipated in view of the sex difference in mean birth weight.

We wish to thank Dr. G. W. Gale, Secretary for Health, Union Health Department, for permission to publish this paper, Dr. S. L. Kark, Medical Officer in Charge, Training Scheme for Health Personnel, Durban, for his constant guidance and constructive criticism, Dr. I. Gordon for his interest and advice, Dr. Sybil Phillips for the extraction of data from records in Capetown, and Mr. L. V. Bradshaw for drawing the Figure. We are indebted to the staffs of the various hospitals who so kindly co-operated in placing their records at our disposal.

Bakwin, H. (1932). Hum. Biol., 4, 1.

\section{REFERENCES}

Bivings, L. (1934). Amer. J. Obstet. Gynec., 27, 725.

Dunham, E. C., Jenss, R. M., and Christie, A. U. (1939). J. Pediat., 14, 156.

Heyns, O. S., and Hersch, S. S. (1944). S. Afr. J. med. Sci., 9, 33.

Jeans, P. C., and Marriott, W. McKim (1947). "Infant Nutrition ", 4th ed., p. 22. Mosby Co., St. Louis.

Le Riche, H. (1938). S. Afr. J. med. Sci., 3, 79.

Meredith, H. V., and Brown, A. W. (1939). Hum. Biol., 11, 24.

Michelson, N. (1943). Amer. J. phys. Anthrop., n.s. 1, 289.

Palmer, C. E., and Ciocco, A. (1945). " Mitchell-Nelson Textbook of Pediatrics ", 4th ed., ed. W. E. Nelson, pp. 14-16. Saunders, Philadelphia.

Stevenson, A. C. (1950). " Recent Advances in Social Medicine", p. 36. Churchill, London.

Union of South Africa (1937). "Report of the Commission of Inquiry regarding the Cape Coloured population of the Union." Government Printer, Pretoria. U.G.54, 1937. 
APPENDIX

Frequency Distribution of Birth Weights

\begin{tabular}{|c|c|c|c|c|c|c|c|c|c|c|c|c|}
\hline \multirow{2}{*}{$\begin{array}{l}\text { Birth } \\
\text { Weight } \\
\text { (lb.) }\end{array}$} & \multicolumn{3}{|c|}{ European } & \multicolumn{3}{|c|}{ Coloured } & \multicolumn{3}{|c|}{ Bantu } & \multicolumn{3}{|c|}{ Indian } \\
\hline & Male & F'male & Total & Male & F'male & Total & Male & F'male & Total & Male & F'male & Total \\
\hline $2-$ & 1 & - & 1 & 2 & 1 & 3 & 5 & 1 & 6 & 2 & - & 2 \\
\hline $2 \frac{1}{2}-$ & - & 1 & 1 & 1 & - & 1 & 6 & 8 & 14 & 1 & 4 & 5 \\
\hline $3-$ & 2 & 1 & 3 & 1 & 5 & 6 & 22 & 30 & 52 & 7 & 8 & 15 \\
\hline $3 \frac{1}{2}-$ & 1 & - & 1 & 4 & 2 & 6 & 29 & 31 & 60 & 7 & 9 & 16 \\
\hline $4-$ & 4 & 5 & 9 & 5 & 4 & 9 & 60 & 68 & 128 & 17 & 29 & 46 \\
\hline $4 \frac{1}{2}-$ & 6 & 13 & 19 & 14 & 9 & 23 & 74 & 105 & 179 & 28 & 36 & 64 \\
\hline $5^{2}-$ & 20 & 19 & 39 & 18 & 23 & 41 & 201 & 239 & 440 & 66 & 104 & 170 \\
\hline $5 \frac{1}{2}-$ & 33 & 48 & 81 & 52 & 49 & 101 & 290 & 377 & 667 & 110 & 108 & 218 \\
\hline $6-$ & 81 & 95 & 176 & 68 & 89 & 157 & 591 & 740 & 1,332 & 183 & 166 & 349 \\
\hline $6 \frac{1}{2}-$ & 99 & 140 & 239 & 94 & 88 & 182 & 717 & 774 & 1,492 & 171 & 153 & 324 \\
\hline $7^{2}-$ & 160 & 161 & 321 & 69 & 84 & 153 & 799 & 644 & 1,443 & 154 & 122 & 277 \\
\hline $7 \frac{1}{2}-$ & 169 & 137 & 306 & 60 & 50 & 110 & 513 & 390 & 903 & 63 & 48 & 111 \\
\hline $8-$ & 154 & 118 & 272 & 42 & 31 & 73 & 301 & 203 & 505 & 49 & 32 & 81 \\
\hline $8 \frac{1}{2}-$ & 83 & 65 & 148 & 20 & 15 & 35 & 169 & 79 & 248 & 24 & 8 & 32 \\
\hline $9-$ & 47 & 32 & 79 & 11 & 5 & 16 & 49 & 39 & 88 & 10 & 5 & 15 \\
\hline $9 \frac{1}{2}-$ & 30 & 11 & 41 & 7 & 2 & 9 & 25 & 14 & 39 & 4 & 3 & 7 \\
\hline $10-$ & 8 & 3 & 11 & 3 & 1 & 4 & 9 & - & 9 & - & 2 & 2 \\
\hline $10 \frac{1}{2}-$ & 2 & 6 & 8 & - & 1 & 1 & 4 & - & 4 & 2 & - & 2 \\
\hline $11-$ & 1 & - & 1 & 一 & - & - & 2 & 一 & 2 & 1 & - & 1 \\
\hline $11 \frac{1}{2}-$ & - & - & - & - & 1 & 1 & - & - & - & - & - & - \\
\hline $12-$ & - & - & 一 & - & - & - & - & - & - & - & 1 & 1 \\
\hline $12 \frac{1}{2}-$ & - & - & - & - & - & - & - & - & 一 & - & - & 一 \\
\hline & 1 & - & 1 & - & - & - & - & - & - & - & 一 & - \\
\hline Total & 902 & 855 & 1,757 & 471 & 460 & 931 & 3,866 & 3,742 & 7,611 & 899 & 838 & 1,738 \\
\hline
\end{tabular}

